\title{
Tuning the phase transition in transition-metal-based magnetocaloric compounds
}

\author{
X. F. Miao, ${ }^{1, *}$ L. Caron, ${ }^{1}$ P. Roy, ${ }^{2}$ N. H. Dung, ${ }^{1}$ L. Zhang, ${ }^{1,3}$ W. A. Kockelmann, ${ }^{4}$ R. A. de Groot,${ }^{2}$ \\ N. H. van Dijk, ${ }^{1}$ and E. Brück ${ }^{1}$ \\ ${ }^{1}$ Fundamental Aspects of Materials and Energy, Faculty of Applied Sciences, Delft University of Technology, Mekelweg 15, \\ 2629 JB Delft, The Netherlands \\ ${ }^{2}$ Electronic Structure of Materials, Faculty of Sciences, Radboud University, 6525 AJ Nijmegen, The Netherlands \\ ${ }^{3}$ BASF Nederland B.V., Strijkviertel 67, 3454 PK De Meern, The Netherlands \\ ${ }^{4}$ ISIS, Rutherford Appleton Laboratory, Chilton, Didcot OX11 OQX, United Kingdom
}

(Received 18 December 2013; revised manuscript received 14 April 2014; published 28 May 2014)

\begin{abstract}
Neutron-diffraction experiments on the $(\mathrm{Mn}, \mathrm{Fe})_{2}(\mathrm{P}, \mathrm{Si})$-type compounds have shown a site preference of $\mathrm{Si}$ atoms in the hexagonal structure. The degree of ordering of $\mathrm{Si}$ depends on the $\mathrm{Si} / \mathrm{P}$ ratio, while it is independent of the $\mathrm{Mn} / \mathrm{Fe}$ ratio. The ferromagnetic-paramagnetic magnetoelastic transition is closely related to the size of the magnetic moment on the $3 f$ site. A preferred occupation of $\mathrm{Si}$ atoms on the $2 c$ site stabilizes and decreases the magnetic moment on the $3 f$ and $3 g$ site, respectively, which is supported by our first-principles density functional theory calculations. This effect, together with the contribution from the Si substitution-induced changes in the interatomic distances, leads to a phase transition that is tunable in temperature and degree of first order in $\mathrm{Mn}_{1.25} \mathrm{Fe}_{0.70} \mathrm{P}_{1-x} \mathrm{Si}_{x}$ compounds. These results provide us with further insight into the relationship between the magnetoelastic phase transition and the local atomic coordination.
\end{abstract}

DOI: 10.1103/PhysRevB.89.174429

PACS number(s): 75.30.Sg, 61.05.fm, 71.15.Mb, 75.30.Kz

\section{INTRODUCTION}

The giant magnetocaloric effect (GMCE), associated with a first-order magnetoelastic transition, makes near-roomtemperature magnetic refrigeration attractive as a highly efficient and environmentally benign cooling technology [1-6]. The evolution of $(\mathrm{Mn}, \mathrm{Fe})_{2}(\mathrm{P}, \mathrm{Si})$ compounds from the $\mathrm{Fe}_{2} \mathrm{P}$ parent phase by partially substituting $\mathrm{Mn}$ for $\mathrm{Fe}$ and $\mathrm{Si}$ for $\mathrm{P}$ brings tunable phase-transition temperatures and GMCE [7-9]. Neutron-diffraction experiments and first-principles calculations indicate the preferential occupation of the two transition-metal atoms in the hexagonal structure (space group $P \overline{6} 2 m)$ [10-13]. Mn prefers the $3 g$ site with five nonmetal nearest neighbors forming a square pyramid, while Fe favors the $3 f$ site surrounded by four nonmetal coordination atoms forming a tetrahedron (see Fig. 1). Strong and weak magnetism appears on the $3 g$ and $3 f$ sites, respectively, as a consequence of the different coordination environments.

The site preference of the nonmetal substitution atoms in $\mathrm{Fe}_{2} \mathrm{P}$ can be estimated based on the atomic radii [14]. Nonmetal atoms with a larger radius than $\mathrm{P}$ (e.g., As and $\mathrm{Si}$ ) are expected to occupy the $2 c$ site, while those having a smaller radius (e.g., B) prefer the $1 b$ site. This prediction has been experimentally confirmed in $\mathrm{Fe}_{2} \mathrm{P}$ by $\mathrm{x}$-ray diffraction in the case of $\mathrm{As}$ substitution [15] and by Mössbauer spectroscopy for B substitution [16]. However, no experimental confirmation of the $\mathrm{Si}$ site preference in $\mathrm{Fe}_{2}(\mathrm{P}, \mathrm{Si})$-type compounds was found.

The first-order magnetoelastic transition in $\mathrm{Fe}_{2} \mathrm{P}$-type compounds is accompanied by changes in the density of states (DOS) of the transition-metal $3 d$ electrons $[9,17,18]$. Mössbauer spectroscopy shows that $\mathrm{Si}$ substitution for $\mathrm{P}$ in $\mathrm{Fe}_{2} \mathrm{P}$ changes the local electronic structure and hyperfine fields of its coplanar $\mathrm{Fe}$ atoms [19]. Theoretical calculations in the $(\mathrm{Mn}, \mathrm{Fe})_{2}(\mathrm{P}, \mathrm{Si})$ yield higher $\mathrm{Fe}(\mathrm{Mn})$ moments

\footnotetext{
*x.f.miao@tudelft.nl
}

when a larger number of coplanar Si nearest neighbors is considered [13]. The interatomic distances also significantly influence the chemical bonding between nearest neighbors, leading to changes in the transition-metal band structure and therefore magnetic moment $[10,18]$. In addition, magnetization measurements reveal that the ferromagnetic(FM)paramagnetic $(\mathrm{PM})$ phase-transition temperature $\left(T_{C}\right)$ increases with increasing $\mathrm{Si}$ content in $(\mathrm{Mn}, \mathrm{Fe})_{2}(\mathrm{P}, \mathrm{Si})$ compounds [8]. This implies that the FM state is stabilized by $\mathrm{Si}$ substitution. Consequently, it is necessary to resolve the underlying relation between the tunable phase transition and the changes in local coordination environment around transition metals in the $\mathrm{Fe}_{2} \mathrm{P}$-type compounds upon nonmetal substitution.

In the present study, we use temperature-dependent neutron diffraction to monitor the evolution of local magnetic moments and interatomic distances across the FM-PM phase transition in $(\mathrm{Mn}, \mathrm{Fe})_{2}(\mathrm{P}, \mathrm{Si})$ compounds. Particular attention is paid to the site preference of $\mathrm{Si}$ atoms and its influence on local magnetic moments. Composition-specific density functional theory (DFT) calculations are performed to verify the experimental results. The influence of $\mathrm{Si}$ substitution on the phase transition is discussed on the basis of $\mathrm{Si}$ site preference and interatomic distances.

\section{EXPERIMENT}

The $\mathrm{Mn}_{1.25} \mathrm{Fe}_{0.70} \mathrm{P}_{1-x} \mathrm{Si}_{x}$ ( $x=0.45,0.50$, and 0.55) compounds were prepared as described by Dung et al. [8,9]. Magnetic characterization was performed using the reciprocating sample option (RSO) mode in a superconducting quantum interference device (SQUID) magnetometer (Quantum Design MPMS 5XL). Calorimetry measurements were performed using a commercial differential scanning calorimeter (TA Instruments Q2000). In situ time-of-flight neutron powder diffraction was measured on the general materials diffractometer (GEM) at the ISIS pulsed neutron source facility, 


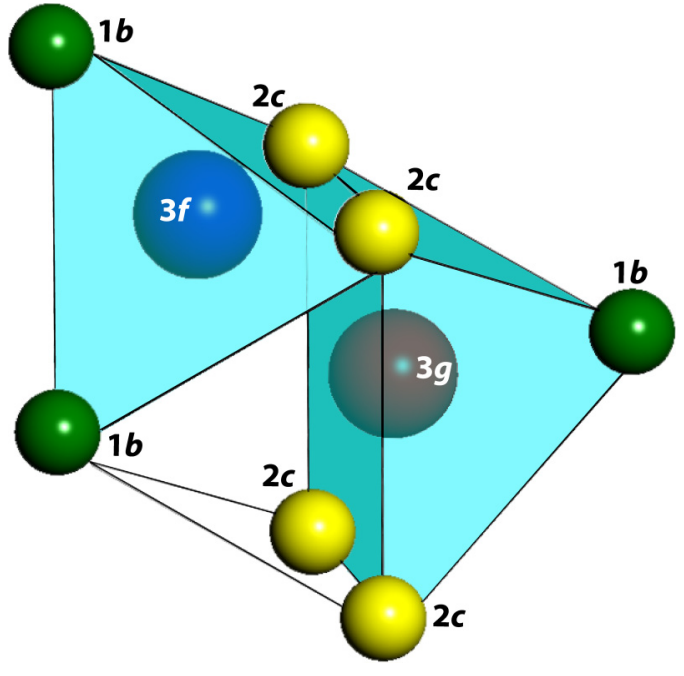

FIG. 1. (Color online) Schematic representation of $(\mathrm{Mn}, \mathrm{Fe})_{2}$ (P,Si) crystal structure.

Rutherford Appleton Laboratory, United Kingdom. The sample as coarse powder (about $7 \mathrm{~g}$ ) was contained in an 8- $\mathrm{mm}$ diameter vanadium can, which was mounted in a cryofurnace. The diffraction data were collected continuously from six banks during the heating process. The heating rate was about $1 \mathrm{~K} / \mathrm{min}$. The temperature ranges were from 10 to $260 \mathrm{~K}$ for the $x=0.45$ sample, from 160 to $300 \mathrm{~K}$ for the $x=0.50$ sample, and from 10 to $375 \mathrm{~K}$ for the $x=0.55$ sample. Nuclear and magnetic structure refinement of the neutron-diffraction patterns was performed using Fullprof's [20] implementation of the Rietveld refinement method.

In the electronic structure calculations, we used the projector augmented wave (PAW) method implemented in the Vienna $A b$ initio Simulation Package (VASP) [21]. Exchange interactions were taken into account using the generalized gradient approximation (GGA) by Perdew, Burke, and Ernzerhof (PBE) [22]. The Brillouin-zone integration was done using a $\gamma$-centered $k$-point mesh of $3 \times 3 \times 8 k$ points in the irreducible part of the Brillouin zone. The cutoff energy of the augmentation function was taken as $500 \mathrm{eV}$ and, for smearing, a Gaussian function was used. For all of the calculations, we relaxed the ionic positions with a force convergence of $0.001 \mathrm{eV} / \AA$ for all of the atoms. The energy convergence criteria was set at $10^{-7} \mathrm{eV}$.

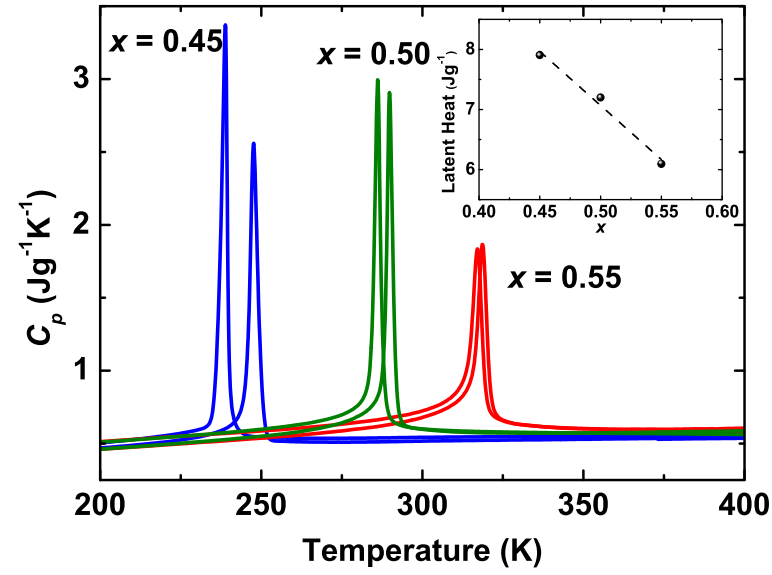

FIG. 3. (Color online) Specific heat as a function of temperature for $\mathrm{Mn}_{1.25} \mathrm{Fe}_{0.70} \mathrm{P}_{1-x} \mathrm{Si}_{x}(x=0.45,0.50$, and 0.55). The latent heat of transition for the three samples is shown in the inset. The dashed line is a guide to the eye.

\section{RESULTS AND DISCUSSIONS}

Temperature-dependent neutron-diffraction patterns are shown in Fig. 2 for the $\mathrm{Mn}_{1.25} \mathrm{Fe}_{0.70} \mathrm{P}_{1-x} \mathrm{Si}_{x}(x=0.45,0.50$, and 0.55 ) compounds. All compounds display a similar evolution of the unit cell parameters with increasing temperature, i.e., a contraction in the $a b$ plane and an expansion along the $c$ axis. Besides, the diffraction peaks show a discontinuity at $T_{C}$. This reveals the first-order nature of the magnetoelastic transition for the $\mathrm{Mn}_{1.25} \mathrm{Fe}_{0.70} \mathrm{P}_{1-x} \mathrm{Si}_{x}$ compounds studied here. With increasing $\mathrm{Si}$ content, $T_{C}$ increases, while the first-order character of the phase transition, manifest in the jump of the peak positions, decreases. This is consistent with magnetization and $\mathrm{x}$-ray diffraction results previously obtained [8]. The specific heat was measured through the phase transition for the three samples. As presented in Fig. 3, the thermal hysteresis is strongly reduced from 7 to $1 \mathrm{~K}$ as $\mathrm{Si}$ content increases from 0.45 to 0.55 , indicating the weakening of the first-order character of the transition. The latent heat also decreases with Si content, reflecting the decrease in the energy barrier associated with the phase transition, in agreement with the thermal hysteresis trend observed. As a result, substituting $\mathrm{Si}$ for $\mathrm{P}$ stabilizes the FM state of $(\mathrm{Mn}, \mathrm{Fe})_{2}(\mathrm{P}, \mathrm{Si})$ compounds, while it weakens the first-order nature of the phase transition.

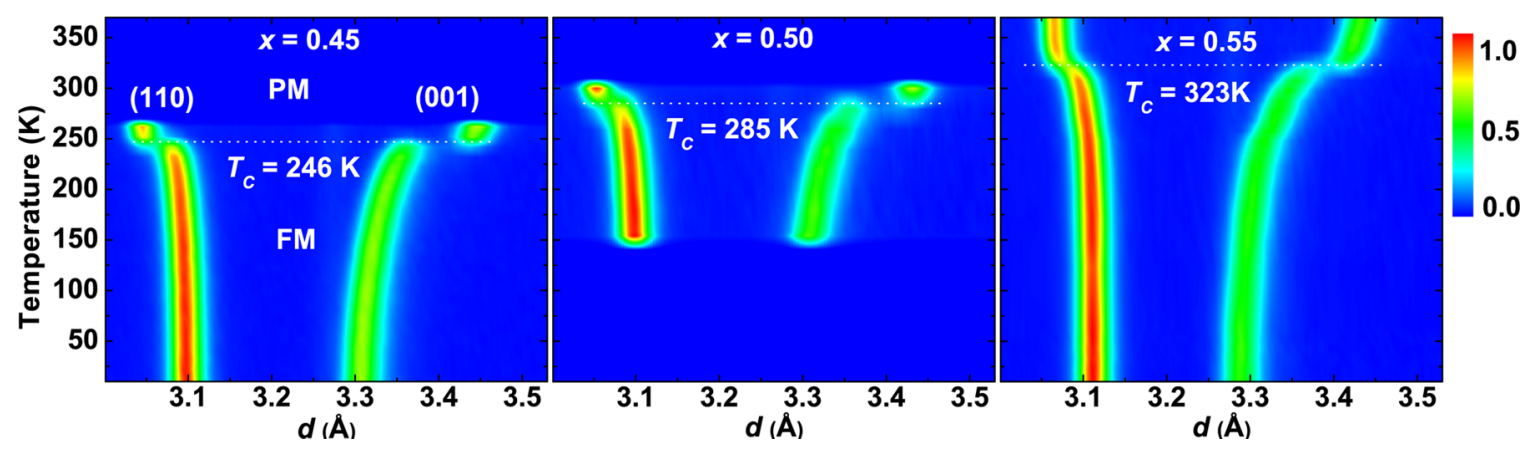

FIG. 2. (Color online) Contour plots of neutron-diffraction patterns for $\mathrm{Mn}_{1.25} \mathrm{Fe}_{0.70} \mathrm{P}_{1-x} \mathrm{Si}_{x}$. The bar on the right represents the normalized intensity scale. 


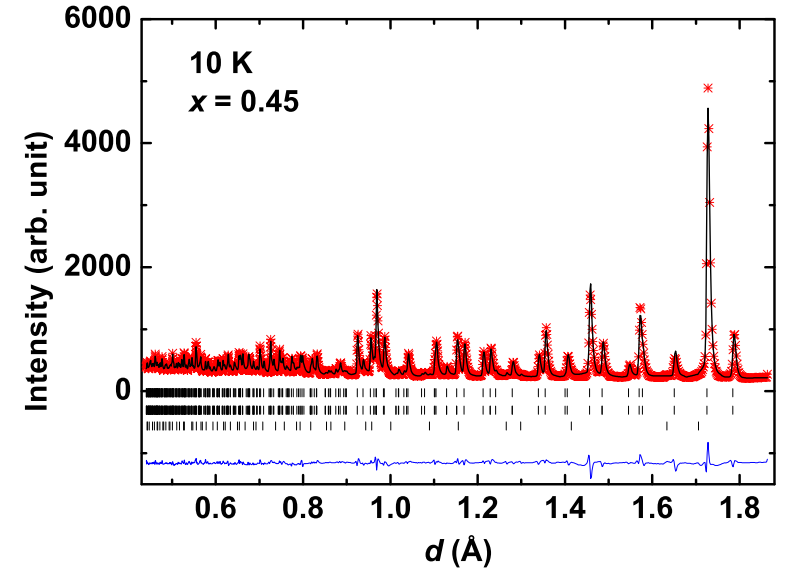

FIG. 4. (Color online) Observed and calculated neutrondiffraction pattern at a scattering angle $(2 \theta)$ of $154.40^{\circ}$ for $\mathrm{Mn}_{1.25} \mathrm{Fe}_{0.70} \mathrm{P}_{0.55} \mathrm{Si}_{0.45}$. Vertical lines indicate the peak positions for the nuclear (top) and magnetic (middle) structure of the main phase, and the impurity phase (Mn,Fe) $)_{3} \mathrm{Si}$ (bottom).

Figure 4 shows the neutron-diffraction pattern of the $x=$ 0.45 compound in the FM state as an example. A good fit is obtained for a hexagonal structure model (space group $P \overline{6} 2 m)$ with magnetic moments within the $a b$ plane. The detailed structure parameters and magnetic moments derived from Rietveld refinements can be seen in Table I. For these Mn-rich samples, the neutron-diffraction results reveal a clear site preference of $\mathrm{Mn}$ and $\mathrm{Fe}$ : the $3 g$ sites are completely occupied by Mn atoms, while $72 \%$ of the $3 f$ sites are taken up by $\mathrm{Fe}$ and $28 \%$ by Mn. The observed site preference of $\mathrm{Mn}$ and $\mathrm{Fe}$ is in good agreement with the literature reports $[10,12,13]$. We further studied the distribution of $\mathrm{Si}$ on the $2 c$ and $1 b$ sites. The fraction of $\mathrm{Si}$ on the $2 c$ site with respect to the total $\mathrm{Si}$ content, hereafter referred to as $f_{2 c}(\mathrm{Si})$, for

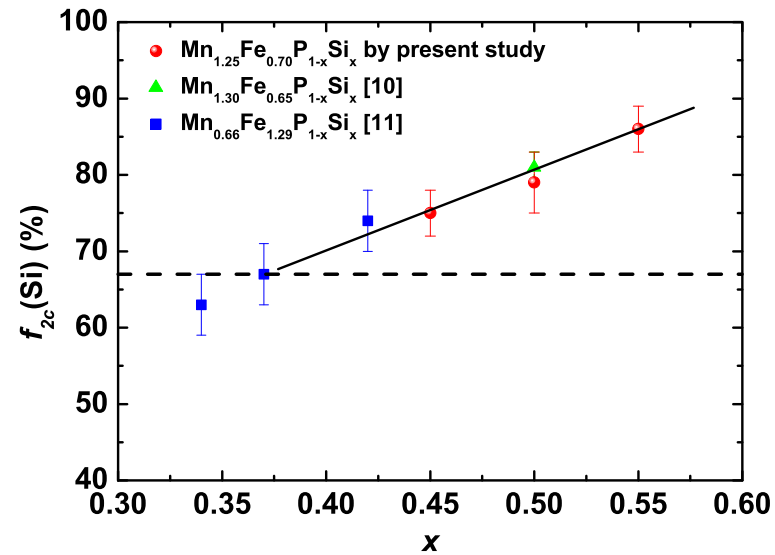

FIG. 5. (Color online) Fraction of $\mathrm{Si}$ on the $2 c$ site in $(\mathrm{Mn}, \mathrm{Fe})_{2}(\mathrm{P}, \mathrm{Si})$ derived from neutron diffraction. The solid line is a guide to the eye. The dashed line indicates the $f_{2 c}(\mathrm{Si})$ in the case of a random Si distribution.

the $\mathrm{Mn}_{1.25} \mathrm{Fe}_{0.70} \mathrm{P}_{1-x} \mathrm{Si}_{x}$ compounds is plotted in Fig. 5. For the present series of compounds, the $f_{2 c}(\mathrm{Si})$ increases linearly with $\mathrm{Si}$ content. All three compounds have a $f_{2 c}(\mathrm{Si})$ higher than expected for a random Si distribution $\left[f_{2 c}(\mathrm{Si}) \approx 67 \%\right]$, indicating a preferred occupation of $\mathrm{Si}$ on the $2 c$ site rather than the $1 b$ site. The $\mathrm{Si}$ site preference is more pronounced in the higher Si-substitution compounds. Interestingly, the $f_{2 c}(\mathrm{Si})$ in $\mathrm{Mn}_{0.66} \mathrm{Fe}_{1.29} \mathrm{P}_{1-x} \mathrm{Si}_{x}(x=0.34,0.37$, and 0.43$)$ compounds [11] and in the $\mathrm{Mn}_{1.30} \mathrm{Fe}_{0.65} \mathrm{P}_{0.50} \mathrm{Si}_{0.50}$ compound [10] also present the same behavior (see Fig. 5). As a result, the $\mathrm{Si}$ distribution is closely related to the $\mathrm{Si}$ content in the compounds, while it is independent of the $\mathrm{Mn} / \mathrm{Fe}$ ratio.

We also explored the $\mathrm{Si}$ distribution by first-principles DFT calculations. A $2 \times 2 \times 1$ supercell is built with lattice parameters $a=6.223 \AA$ and $c=3.289 \AA$ taken from the

TABLE I. Structural parameters of $\mathrm{Mn}_{1.25} \mathrm{Fe}_{0.70} \mathrm{P}_{1-x} \mathrm{Si}_{x}(x=0.45,0.50$, and 0.55) in the FM state. Space group: $P \overline{6} 2 m$. Atomic positions: $3 f\left(x_{1}, 0,0\right), 3 g\left(x_{2}, 0,1 / 2\right), 2 c(1 / 3,2 / 3,0)$, and $1 b(0,0,1 / 2)$. Magnetic moments: within $a b$ plane.

\begin{tabular}{|c|c|c|c|c|}
\hline & Parameters & $x=0.45(10 \mathrm{~K})$ & $x=0.50(160 \mathrm{~K})$ & $x=0.55(10 \mathrm{~K})$ \\
\hline \multirow[t]{3}{*}{ Unit cell } & $a$ & $6.1938(1)$ & $6.1963(2)$ & $6.2226(1)$ \\
\hline & $c$ & $3.3063(1)$ & $3.3091(2)$ & $3.2894(1)$ \\
\hline & $V\left(\AA^{3}\right)$ & $109.845(4)$ & $110.031(5)$ & $110.303(5)$ \\
\hline \multirow[t]{4}{*}{$3 f$} & $x_{1}$ & $0.2584(3)$ & $0.2582(5)$ & $0.2593(3)$ \\
\hline & $n(\mathrm{Fe}) / n(\mathrm{Mn})$ & $0.181 / 0.069(1)$ & $0.177 / 0.073(1)$ & $0.178 / 0.072(1)$ \\
\hline & $M\left(\mu_{B}\right)$ & $1.48(8)$ & $1.42(12)$ & $1.57(11)$ \\
\hline & $B\left(\AA^{2}\right)$ & $0.30(4)$ & $0.35(6)$ & $0.33(5)$ \\
\hline \multirow[t]{4}{*}{$3 g$} & $x_{2}$ & $0.5965(5)$ & $0.5962(7)$ & $0.5968(6)$ \\
\hline & $n(\mathrm{Mn}) / n(\mathrm{Fe})$ & $0.250 / 0$ & $0.250 / 0$ & $0.250 / 0$ \\
\hline & $M\left(\mu_{B}\right)$ & $2.59(8)$ & $2.30(11)$ & $2.30(9)$ \\
\hline & $B\left(\AA^{2}\right)$ & $0.29(6)$ & $0.62(8)$ & $0.42(7)$ \\
\hline \multirow[t]{2}{*}{$2 c$} & $n(\mathrm{P}) / n(\mathrm{Si})$ & $0.082 / 0.085(4)$ & $0.068 / 0.098(5)$ & $0.048 / 0.118(4)$ \\
\hline & $B\left(\AA^{2}\right)$ & $0.17(6)$ & $0.41(9)$ & $0.26(9)$ \\
\hline \multirow[t]{5}{*}{$1 b$} & $n(\mathrm{P}) / n(\mathrm{Si})$ & $0.056 / 0.028(4)$ & $0.057 / 0.027(5)$ & $0.064 / 0.019(4)$ \\
\hline & $B\left(\AA^{2}\right)$ & $0.10(9)$ & $0.31(13)$ & $0.21(11)$ \\
\hline & $R p(\%)$ & 3.61 & 3.65 & 4.08 \\
\hline & $w R p(\%)$ & 4.91 & 4.84 & 5.03 \\
\hline & $\chi^{2}$ & 6.63 & 7.57 & 7.40 \\
\hline
\end{tabular}




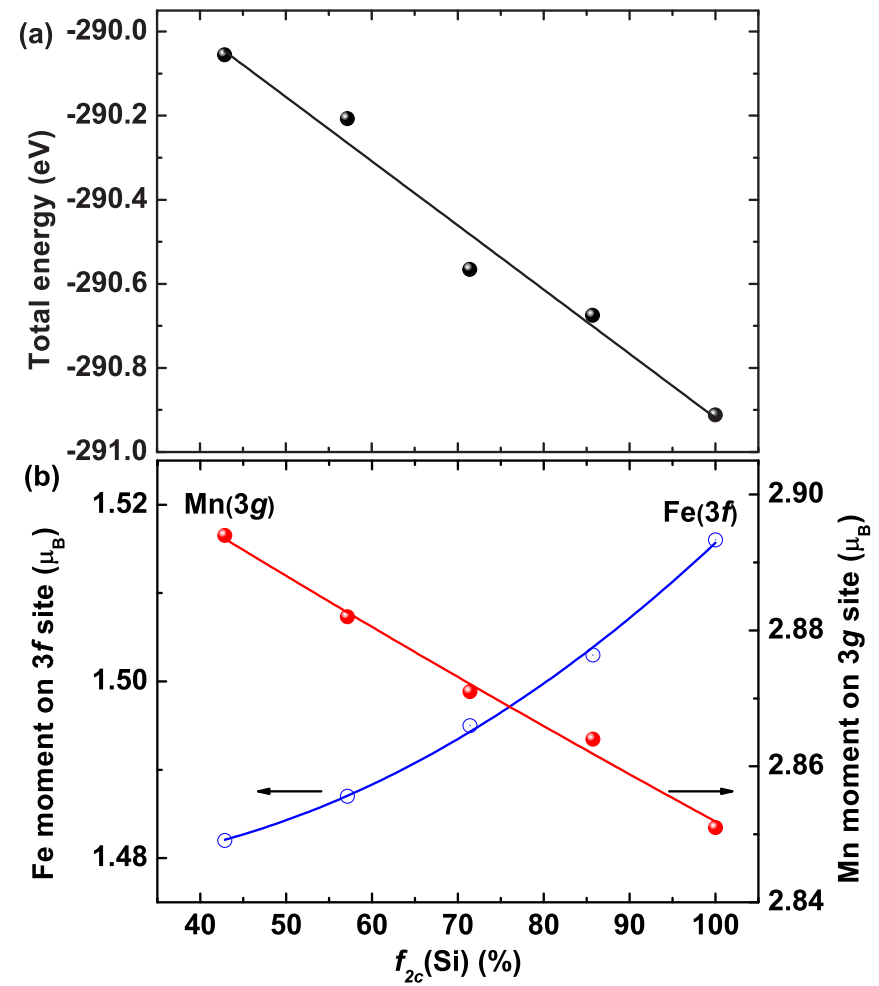

FIG. 6. (Color online) The influence of $\mathrm{Si}$ distribution on (a) total energy and (b) sublattice magnetic moments for $\mathrm{Mn}_{1.25} \mathrm{Fe}_{0.75} \mathrm{P}_{0.42} \mathrm{Si}_{0.58}$ from first-principles DFT calculations. The solid lines are guides to the eye.

neutron-diffraction experiments for $x=0.55$. The supercell contains $15 \mathrm{Mn}, 9 \mathrm{Fe}, 7 \mathrm{Si}$, and $5 \mathrm{P}$ atoms, with a composition $\mathrm{Mn}_{1.25} \mathrm{Fe}_{0.75} \mathrm{P}_{0.42} \mathrm{Si}_{0.58}$ close to the $x=0.55$ sample. Atomic configurations for different $\mathrm{Si}$ distributions on the $2 c$ and $1 b$ sites are modeled by filling the eight $2 c$ sites in the supercell with a different number (3-7) of $\mathrm{Si}$ atoms. The corresponding $f_{2 c}(\mathrm{Si})$ are $3 / 7,4 / 7,5 / 7,6 / 7$, and 1 . The total energy as a function of the $f_{2 c}(\mathrm{Si})$ is presented in Fig. 6(a). The total energy decreases with increasing $f_{2 c}(\mathrm{Si})$. This clearly reveals that Si prefers the $2 c$ sites instead of the $1 b$ sites, in good agreement with our experimental results and previous theoretical calculation reports [13,23].

The site preference of $\mathrm{Si}$ on the $2 c$ site can be understood by the nonmetal atomic size factor proposed by Rundqvist [14] since Si has a larger atomic radius than P. However, this size factor is not observed in the case of As substitution in the hexagonal $(\mathrm{Mn}, \mathrm{Fe})_{2} \mathrm{P}_{1-x} \mathrm{As}_{x}$ compounds. The substituted As atoms are statistically distributed on the $2 c$ and $1 b$ sites [24], although the atomic radius of As is much larger than that of P. The strong contrast of the site occupation between As and Si likely arises from their different chemical properties. As and $\mathrm{P}$ have the same number of valence electrons, while Si has less. Therefore, while As substitution has no effect on the electronic environment, $\mathrm{Si}$ induces a significant electronic reconfiguration. As a result, $\mathrm{Si}$ atoms prefer to occupy the lower-energy $2 c$ site.

Figure 7 displays the thermal evolution of the sublattice magnetic moments derived from neutron-diffraction measurements for $\mathrm{Mn}_{1.25} \mathrm{Fe}_{0.70} \mathrm{P}_{1-x} \mathrm{Si}_{x}$ compounds. The magnetic moment on the $3 f$ site shows an increasing trend with

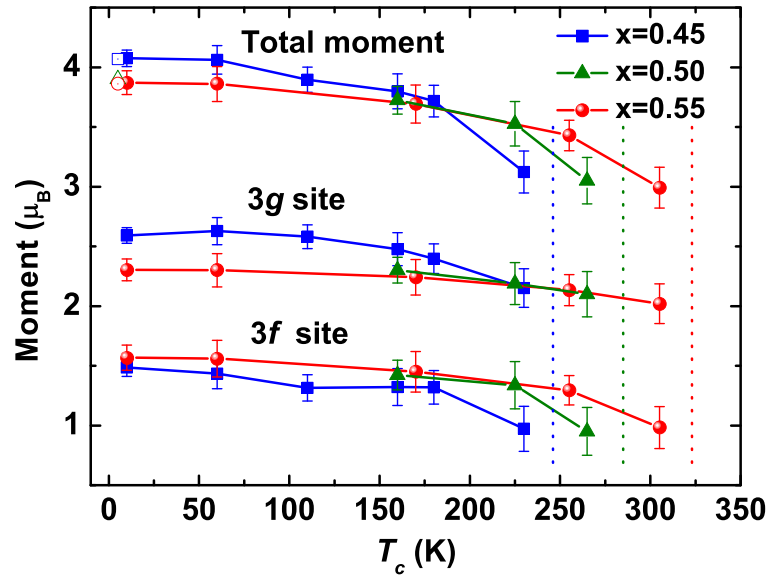

FIG. 7. (Color online) Temperature dependence of magnetic moments derived from neutron diffraction for $\mathrm{Mn}_{1.25} \mathrm{Fe}_{0.70} \mathrm{P}_{1-x} \mathrm{Si}_{x}$. Total magnetic moments from magnetization measurements at $5 \mathrm{~K}$ are also shown with the open symbols. The dotted lines indicate the position of $T_{C}$ for each sample.

increasing $\mathrm{Si}$ content, while the moment on the $3 g$ site shows the opposite trend. Since the moment in the $3 g$ site is roughly double that of the $3 f$ site, the total moment decreases with increasing Si substitution, which is consistent with our magnetization measurements. All compounds display a similar temperature dependence of the magnetic moments on the $3 f$ and $3 g$ sites. The moment on the $3 g$ site is hardly influenced by increasing temperature, whereas the moment on the $3 f$ site drops with increasing temperature, particularly in the vicinity of $T_{C}$. Besides that, our theoretical calculations [9] indicate that $\mathrm{Fe}$ on the $3 f$ site almost loses all the magnetic moment after the ferromagnetic-paramagnetic magnetoelastic transition, while $\mathrm{Mn}$ on the $3 g$ site still carries a magnetic moment of over $2 \mu_{B}$. These results demonstrate that the magnetoelastic transition in $\mathrm{Mn}_{1.25} \mathrm{Fe}_{0.70} \mathrm{P}_{1-x} \mathrm{Si}_{x}$ compounds is closely linked with the decay of the magnetic moment on the $3 f$ site. However, the high-moment state of the $3 f$ site persists to higher temperatures for the compounds containing more $\mathrm{Si}$ atoms. This gives us clear evidence of the enhancement of the FM state by $\mathrm{Si}$ substitution for $\mathrm{P}$ in $\mathrm{Mn}_{1.25} \mathrm{Fe}_{0.70} \mathrm{P}_{1-x} \mathrm{Si}_{x}$ compounds. Therefore, the rise in $T_{C}$ of the FM-PM transition with increasing Si content (see Fig. 2) can be attributed to the stabilization of the magnetic moment on the $3 f$ site.

The first-order phase-transition behavior in the $(\mathrm{Mn}, \mathrm{Fe})_{2}(\mathrm{P}, \mathrm{Si})$-type compounds is closely related to the unique mixed magnetism of the system $[9,10]$. The preferred occupation of $\mathrm{Si}$ on the $2 c$ site causes significant electronic reconfiguration around the $3 f$ and $3 g$ sites, which stabilizes and decreases the magnetic moment on the $3 f$ and $3 g$ site, respectively. This weakens the itinerant character of the moments on the $3 f$ site and the localized character on the $3 g$ site. Thus, by changing $\mathrm{Si}$ content, the electronic structure is changed, allowing us to effectively tune the mixed magnetism in the system and the phase-transition character. Consequently, the site preference of $\mathrm{Si}$ allows us to tune the order of the phase transition, while the As atom, having the same number of valence electrons as $\mathrm{P}$ and thus being statistically distributed, cannot $[25,26]$. 
The Fe (Mn) magnetic-moment formation is in competition with chemical bonding in the $\mathrm{Mn}_{1.25} \mathrm{Fe}_{0.70} \mathrm{P}_{1-x} \mathrm{Si}_{x}$ compounds, which is strongly influenced by the coordination environment around the Fe (Mn) atoms. Two main contributions from the coordination atoms should be taken into consideration: the atomic species and the interatomic distances. Figure 6(b) illustrates the magnetic moments on the $3 f$ and $3 g$ sites as a function of $f_{2 c}(\mathrm{Si})$ obtained from our DFT calculations. Higher and lower magnetic moments are predicted on the $3 f$ and $3 g$ sites, respectively, when more Si atoms occupy the $2 c$ sites. According to the electronic structure calculations, $(\mathrm{Mn}, \mathrm{Fe})_{2}(\mathrm{P}, \mathrm{Si})$ has only $\mathrm{Fe}$ and $\mathrm{Mn} d$ electrons close to the Fermi level, while $\mathrm{P}$ and $\mathrm{Si} p$ electrons are located far below [13]. Electron transfer from the Fe and $\mathrm{Mn} d$ band to the $\mathrm{P}$ and Si $p$ band would be expected to fill the P and Si $p$ band, which enhances the splitting of the Fe and Mn $d$ band and thus increases the magnetic moment of the Fe and Mn supplying the electrons. This electron transfer feature is more pronounced for coplanar metal-nonmetal neighbors, as indicated by the isomer shift in Mössbauer spectra of $\mathrm{Fe}_{2}(\mathrm{P}, \mathrm{Si})$ compounds [19]. Since Si has less valence electrons than P, the preferred occupation of $\mathrm{Si}$ on the $2 c$ site would bring larger magnetic moments on its coplanar $3 f$ site. These results suggest that larger magnetic moments will develop on the $3 f$ or $3 g$ site when there are more coplanar Si nearest neighbors, which is in accordance with previous theoretical calculation results [13].

The interatomic distance also plays a crucial role in the competition between the magnetic moment and chemical

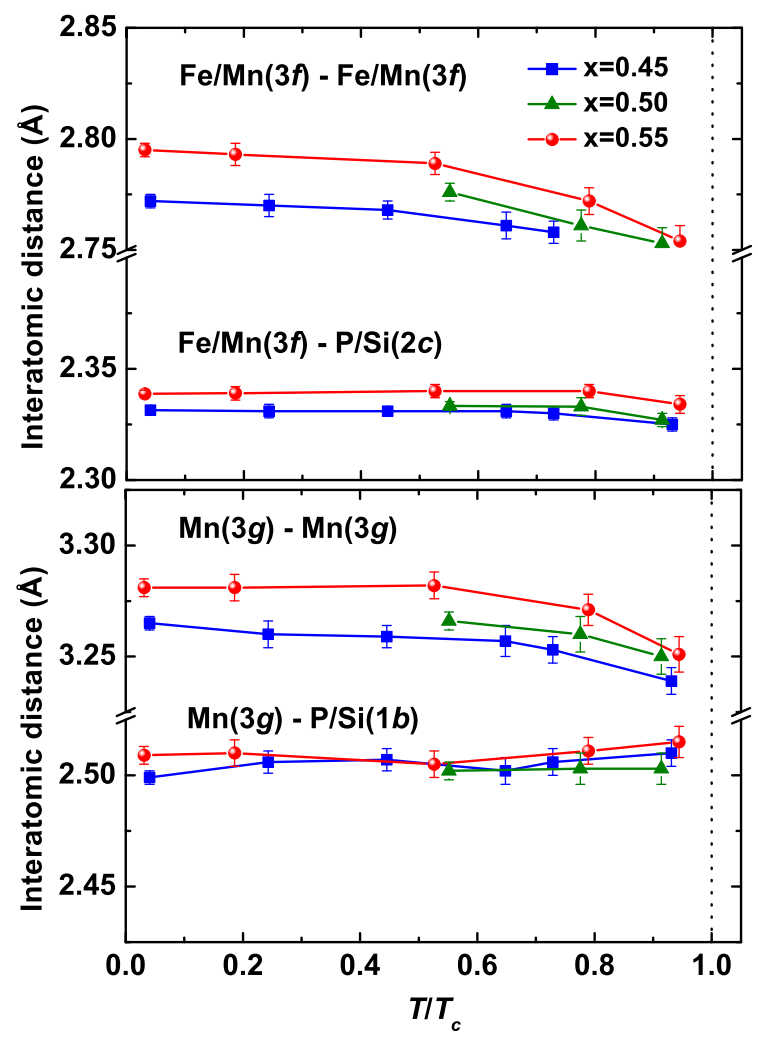

FIG. 8. (Color online) Intralayer interatomic distances as a function of temperature derived from neutron diffraction for $\mathrm{Mn}_{1.25} \mathrm{Fe}_{0.70} \mathrm{P}_{1-x} \mathrm{Si}_{x}$. Dotted lines indicate the position of the normalized $T_{C}$. bonding. Our neutron-diffraction experiments reveal that compared with the interlayer interatomic distances, the intralayer interatomic distances show a much stronger dependence on temperature or $\mathrm{Si}$ substitution. This probably arises from the reconfiguration of electronic structure during the phase transition or upon $\mathrm{Si}$ substitution taking place mainly within the layer. The intralayer interatomic distances decrease with rising temperature, as shown in Fig. 8. As a result, the magnetic moments decrease with increasing temperature due to the growing overlap of $3 d$ states as well as the enhanced metal-nonmetal hybridization (see Fig. 7). Moreover, the intralayer distances expand when more $\mathrm{Si}$ atoms replace $\mathrm{P}$ (see Fig. 8). Larger magnetic moments, therefore, are expected on the $3 f$ and $3 g$ sites for the higher Si-containing compounds. This is confirmed by the neutron-diffraction results for the magnetic moment on the $3 f$ site (see Fig. 7). However, the observed magnetic moment on the $3 g$ site shows an unexpected decrease with increasing Si substitution, although the $\mathrm{Mn}(3 g)-\mathrm{Mn}(3 g)$ distance expands. Such a deviation can be understood by the increasing $\mathrm{Si}$ occupancy on the $2 c$ site with increasing Si content, as indicated in Fig. 5. In the higher Si-containing samples, less electron transfer occurs between the $3 g-1 b$ coplanar sites and it therefore causes less moment to develop on the $3 g$ site [see Fig. 6(b)]. As a consequence, the changes in the sublattice magnetic moments induced by $\mathrm{Si}$ substitution are the results of both Si site preference and the varying interatomic distances.

\section{CONCLUSIONS}

In summary, the local magnetic and nuclear structure in $\mathrm{Mn}_{1.25} \mathrm{Fe}_{0.70} \mathrm{P}_{1-x} \mathrm{Si}_{x}(x=0.45,0.50$, and 0.55$)$ compounds has been monitored across the magnetoelastic transition using temperature-dependent neutron diffraction. The Si distribution on the $2 c$ and $1 b$ sites in the hexagonal $(\mathrm{Mn}, \mathrm{Fe})_{2}(\mathrm{P}, \mathrm{Si})$-type compounds can be predicted based on our neutron-diffraction results, which is dependent on the $\mathrm{Si} / \mathrm{P}$ ratio while independent of the $\mathrm{Mn} / \mathrm{Fe}$ ratio. The FM-PM magnetoelastic transition is accompanied by the decay of the magnetic moment on the $3 f$ site. The clear preference of $\mathrm{Si}$ to occupy the $2 c$ site stabilizes and decreases the magnetic moment on the $3 f$ and $3 g$ site, respectively, which is supported by our first-principles calculations. The intralayer interatomic distances are expanded by $\mathrm{Si}$ substitution, which also enhances the magnetic moments on the $3 f$ sites. The phase transition with tunable degree of first order and critical temperature in $(\mathrm{Mn}, \mathrm{Fe})_{2}(\mathrm{P}, \mathrm{Si})$-type compounds is attributed to the observed Si site preference in combination with changing interatomic distances. Increasing $\mathrm{Si}$ content weakens the mixed magnetism in $(\mathrm{Mn}, \mathrm{Fe})_{2}(\mathrm{P}, \mathrm{Si})$ type compounds, inducing normal ferromagnetic behavior accompanied by second-order phase transitions.

\section{ACKNOWLEDGMENTS}

The authors would like to thank Anton J. E. Lefering and Bert Zwart for their help in sample preparation, and acknowledge the assistance of Ron Smith during the neutrondiffraction experiments at ISIS. This work is part of the Industrial Partnership Program of the Dutch Foundation for Fundamental Research on Matter (FOM), and co-financed by BASF New Business. 
[1] X. X. Zhang, J. Tejada, Y. Xin, G. F. Sun, K. W. Wong, and X. Bohigas, Appl. Phys. Lett. 69, 3596 (1996).

[2] V. K. Pecharsky and K. A. Gschneidner, Jr., Phys. Rev. Lett. 78, 4494 (1997).

[3] H. Wada and Y. Tanabe, Appl. Phys. Lett. 79, 3302 (2001).

[4] O. Tegus, E. Brück, K. H. J. Buschow, and F. R. de Boer, Nature (London) 415, 150 (2002).

[5] A. Fujita, S. Fujieda, Y. Hasegawa, and K. Fukamichi, Phys. Rev. B 67, 104416 (2003).

[6] E. Brück, J. Phys. D: Appl. Phys. 38, R381 (2005).

[7] N. H. Dung, L. Zhang, Z. Q. Ou, and E. Brück, Appl. Phys. Lett. 99, 092511 (2011).

[8] N. H. Dung, L. Zhang, Z. Q. Ou, and E. Brück, Scr. Mater. 67, 975 (2012).

[9] N. H. Dung, Z. Q. Ou, L. Caron, L. Zhang, D. T. Cam Thanh, G. A. de Wijs, R. A. de Groot, K. H. J. Buschow, and E. Brück, Adv. Energy Mater. 1, 1215 (2011).

[10] N. H. Dung, L. Zhang, Z. Q. Ou, L. Zhao, L. van Eijck, A. M. Mulders, M. Avdeev, E. Suard, N. H. van Dijk, and E. Brück, Phys. Rev. B 86, 045134 (2012).

[11] Z. Q. Ou, L. Zhang, N. H. Dung, L. van Eijck, A. M. Mulders, M. Avdeev, N. H. van Dijk, and E. Brück, J. Magn. Magn. Mater. 340, 80 (2013).

[12] M. Hudl, P. Nordblad, T. Björkman, O. Eriksson, L. Häggström, M. Sahlberg, Y. Andersson, E. K. Delczeg-Czirjak, and L. Vitos, Phys. Rev. B 83, 134420 (2011).

[13] X. B. Liu and Z. Altounian, J. Appl. Phys. 105, 07 A902 (2009).
[14] S. Rundqvist, Arkiv för Kemi 20, 67 (1962).

[15] A. Catalano, R. J. Arnott, and A. Wold, J. Solid State Chem. 7, 262 (1973).

[16] R. Chandra, S. Bjarman, T. Ericsson, L. Häggström, C. Wilkinson, and R. Wäppling, J. Solid State Chem. 34, 389 (1980).

[17] X. B. Liu, J. Ping Liu, Q. Zhang, and Z. Altounian, Phys. Lett. A 377, 731 (2013).

[18] Z. Gercsi, E. K. Delczeg-Czirjak, L. Vitos, A. S. Wills, A. Daoud-Aladine, and K. G. Sandeman, Phys. Rev. B 88, 024417 (2013).

[19] P. Jernberg, A. A. Yousif, L. Häggström, and Y. Andersson, J. Solid State Chem. 53, 313 (1984).

[20] J. Rodríguez-Carvajal, Physica B 192, 55 (1993).

[21] G. Kresse and J. Furthmüller, Phys. Rev. B 54, 11169 (1996).

[22] J. P. Perdew, K. Burke, and M. Ernzerhof, Phys. Rev. Lett. 77, 3865 (1996).

[23] E. K. Delczeg-Czirjak, L. Delczeg, M. P. J. Punkkinen, B. Johansson, O. Eriksson, and L. Vitos, Phys. Rev. B 82, 085103 (2010).

[24] M. Bacmann, J. L. Soubeyroux, R. Barrett, D. Fruchart, R. Zach, S. Niziol, and R. Fruchart, J. Magn. Magn. Mater. 134, 59 (1994).

[25] R. P. Hermann, O. Tegus, E. Brück, K. H. J. Buschow, F. R. de Boer, G. J. Long, and F. Grandjean, Phys. Rev. B 70, 214425 (2004).

[26] O. Beckman and L. Lundgren, in Handbook of Magnetic Materials., edited by K. H. J. Buschow (North-Holland, Amsterdam, 1991), Vol. 6, pp. 181-287. 